\title{
Non-Hodgkin's Lymphoma in Pregnancy: A Case Report
}

\author{
ASMAHABIB $^{1}$, MD. MOFAZZEL HOSSAIN ${ }^{2}$
}

\begin{abstract}
:
Non-Hodgkin lymphoma with concurrent pregnancy produces management dilemma regarding obstetrical aspect and chemoradiation for the disease itself. Successful pregnancy outcome depends upon several factors. Stage of Non-Hodgkin Lymphoma at presentation, gestational age at the time of diagnosis, response to chemotherapy, presence of compression symptoms or systemic B symptoms, concomitant obstetric complications emerging during antenatal follow-up, teratogenic /developmental defects of the unborn baby and its sequel, cardiotoxicity of Doxorubicin in particular are among the various factors affecting management and prognosis of each case. This case report delineates the management of a patient diagnosed as NonHodgkin's lymphoma at 18 weeks of gestation with a successful foetal outcome and ultimate demise of the patient from progressive disease eight months after delivery.
\end{abstract}

\section{Introduction:}

The incidence of Non- Hodgkin lymphoma during pregnancy is rare, with fewer than one hundred cases reported..$^{1}$ Most Non-Hodgkin lymphomas that occur during pregnancy are aggressive and delay of therapy until after delivery appears to have poor outcomes according to anecdotal case series. Consequently, some investigators favor immediate therapy, even during pregnancy. Termination of pregnancy in the first trimester may be an option to allow chemotherapy with or without radiation therapy for women with aggressive NHL. During the second and third trimester of pregnancy early delivery when feasible may minimize or avoid exposure of the fetus to chemotherapy or radiation therapy. When possible, treatment should be postponed until after an early delivery. Women with indolent (slow-growing) NonHodgkin lymphoma can usually delay treatment during pregnancy with watchful waiting unless there are clear indications for treatment, such as: local symptoms due to progressive or bulky nodal disease, compromise of normal organ function due to progressive or bulky disease, presence of systemic B symptoms, presence of symptomatic extranodal disease, such as effusions, cytopenias due to extensive bone marrow infiltration, autoimmune hemolytic anemia or thrombocytopenia, or hypersplenism and an increase in disease tempo. ${ }^{2}$

\section{Case report}

A 26 year old woman Para 1 (normal vaginal delivery, age of the child 6 years) presented in the department of Medicine of Bangladesh Medical College and Hospital on 30/03/08 with the complaints of shortness of breath and cough for 7 days, pain in the right side of the back of the chest for 10 days and pregnancy for 16 weeks. She was a non-smoker and had no prior history of tuberculosis or bronchial asthma. Chest Xray P/A view (Figure: $1 \mathrm{a}, \mathrm{b}$ ) and Ultrasonograph of chest revealed collapse consolidation/encysted pneumothorax in the right hemithorax. Ultrasonography guided Fine Needle Aspiration Cytology (FNAC) of Right lung lesion on 11/4/08 revealed Non-Hodgkin Lymphoma, Intermediate grade. Description showed highly cellular smear with plenty of small round cells, disposed singly or in small groups having round nucleus with very scanty cytoplasm, some of the cells contain nuclear indentation. Immunophenotyping to determine the type of neoplasm was not done due to non-affordability of the patient. Bone marrow was not done as the stage of $\mathrm{NHL}$ was already Stage IV at the time of presentation due to diffuse involvement of lung. Though her husband gave written consent for termination of pregnancy, she refused to terminate. She received the first cycle of chemotherapy with the CHOP regimen (Cyclophosphamide 800mg, Doxorubicin 70 mg,

1. Resident Assistant Professor, Department of Obstetrics and Gynaecology, Bangladesh Medical College and Hospital, Dhanmondi, Dhaka

2. Medical Oncology, Professor of Oncology, Bangladesh Medical College and Hospital, Dhanmondi, Dhaka 


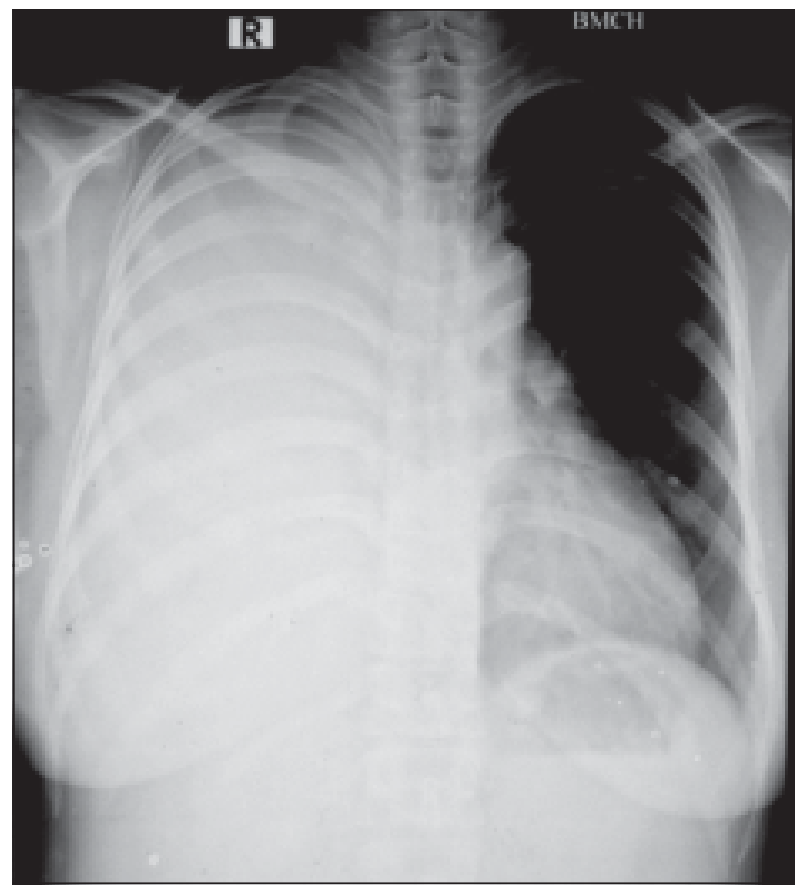

(a)

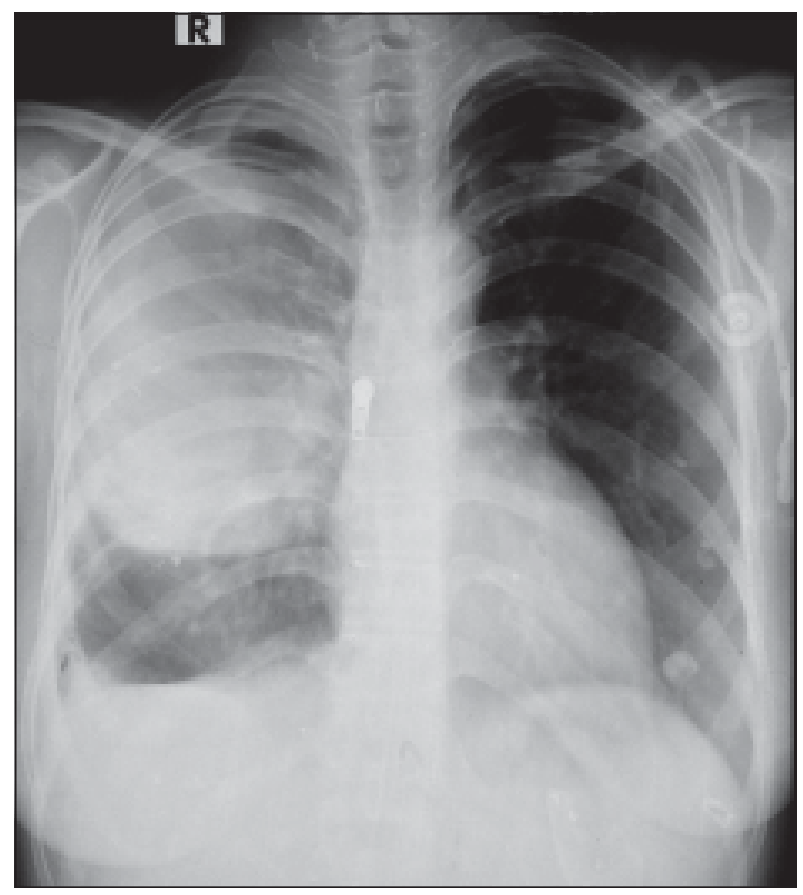

(b)

Fig.-1 (a, b): X-ray chest showing collapse consolidation of the right lung field.

Vincristine $1.6 \mathrm{mg}$ and Prednisolone $80 \mathrm{mg}$ at 17 weeks of pregnancy. She weighed $54 \mathrm{Kg}$.

Her pregnancy was monitored both clinically and by ultrasonography. Anomaly scan at $18+$ weeks revealed no detectable congenital anomaly. She received four more cycles of $\mathrm{CHOP}$ chemotherapy at approximately 3 weeks interval at 21 weeks (02/05/08), 24 weeks (27/05/08), 28 weeks (22/06/08) and 32 weeks (26/ $08 / 08$ ) of pregnancy. During chemotherapy, hematological parameters were normal, except $\mathrm{Hb}$ $8.94 \mathrm{gm} / \mathrm{dl}$ after the third chemotherapy, it was managed by transfusion of 1 unit of packed cell transfusion. Her liver function was normal except for ALT which was $87 \mathrm{U} / \mathrm{L}$. She had persistent cough and intermittent chest pain during the whole pregnancy period. Her pregnancy was uneventful up to 34 weeks of pregnancy when she had aggravation of burning chest pain which persisted for 3 weeks and was managed conservatively. Echocardiography revealed normal left ventricular function with a $64 \%$ ejection fraction at rest. At 37 completed weeks she had lower uterine caesarean section after failed induction and she delivered a healthy female of $2.75 \mathrm{~kg}$ with good APGAR score. The baby was screened for haematological parameters, infection and anomaly and was found to be normal by the Paediatric department. Computerized tomograghic scan of the lung (Figure : 2) was done to evaluate her postchemotherapy state which revealed huge right sided pleural effusion with nearly complete atelectasis of right lung with diffuse hypodense areas suggesting infiltration of the right lung or necrosis after contrast enhancement with patchy parenchymal densities in left lower lung and compression of the mediastinum, trachea and both principal bronchus, with enlarged mediastinal and hilar lymph nodes. Pleurocentesis



Fig.-2: Computed tomography scan of the lung showing diffuse hypodense areas in the right lung field. 
was done which demonstrated lymphocytic predominance (90\%) with RBC, raised LDH (568 U/ $\mathrm{L})$, negative for malignant cells, but few reactive mesothelial cells. She received the $6^{\text {th }}$ cycle of chemotherapy 10 days after delivery. Her haematological parameters showed bicytopenia with $\mathrm{Hb} \% 7.18 \mathrm{gm} / \mathrm{dl}$ and platelet count 80,000/L for which she received two units of blood transfusion. USG guided FNAC was repeated from the left lung lesion which showed Non-Hodgkin Lymphoma ( Small round cell tumour; many small atypical cells arranged in sheets with round nucleus and scanty cytoplasm, some showing plasmacytoid appearance). For intermittent but persistent chest pain and respiratory embarrassment, she was referred to the Radiotherapy department of Dhaka Medical College and Hospital for Chest Radiotherapy after another cycle of CHOP chemotherapy on 08/11/08. She received accelerated hyperfractionated thoracic radiotherapy (36 Gy TeleCobalt 60 in right anterior and posterior chest) in 18 fractions over 5 weeks from 18/11/08 to 24/12/08. Subsequently her clinical performance declined along with haematological status and serological parameters (LDH $493 \mathrm{U} / \mathrm{L}$, serum albumin $35 \mathrm{gm} / \mathrm{l}$, rising alkaline phosphatase and serum creatinine 0.6 to $1.1 \mathrm{mg} / \mathrm{dl}$ ). USG of the whole abdomen 2 months postRadiotherapy revealed normal findings. She received symptomatic treatment for chest pain with analgesics and gabapentin. She died approximately one year after diagnosis that is eight months following delivery from acute respiratory failure due to progressive disease. The child is in apparent good health, now 11 months of age deprived from breast milk as per the strict instruction of the pediatricians to avoid the effect of chemoradiation.

\section{Discussion:}

The prognosis, survival and response of NHL to therapy are related to the histological variant. According to the most accepted classification of Non -Hodgkin's Lymphoma proposed by Rappaport, except for the well-differentiated lymphocytic variety, the diffuse types have a poorer prognosis than nodular ones. 3 Aggressive lymphomas also called intermediate-grade and high-grade lymphomas grow and spread quickly and are usually associated with severe symptoms. Lymphoblastic lymphoma, diffuse small noncleaved cell lymphoma/Burkitt lymphoma, and mantle cell lymphoma are 3 types of aggressive adult non-Hodgkin lymphoma. Aggressive lymphomas are seen more frequently in patients who are HIV-positive (AIDSrelated Iymphoma), in patients who are on immunosuppressant therapy after organ transplantation or those who have been treated previously for Hodgkin lymphoma or in the presence of inherited immune disorders (for example, hypogammaglobulinemia or Wiskott-Aldrich syndrome). ${ }^{4}$ In our case no tests were done to find out the possible cause of development of NonHodgkin's Lymphoma, but pregnancy itself is a state of immunosuppression.

The differential diagnosis of aggressive small round cell non-cleaved non-Hodgkins lymphoma of the lung presenting with hilar and mediastinal lymphadenopathy are other small round cell tumors of this region: (1) Small cell mesothelioma: Primary mesothelioma is extremely rare in children and young adults. (2) primitive neuroectodermal tumor (PNET)/ Ewing's sarcoma family of tumors. The age of presentation is similar. Histologically, they are composed of small round, undifferentiated blue cells with scant cytoplasm. Immunohistologically, PNET/ Ewing's sarcomas usually show a unidirectional differentiation toward neural elements. (3) DSRCT ( Desmoplastic small round cell tumour): The age and clinical presentation is similar; chest pain and pleural effusion in young patients with a male predominance. Histologically, angulated nests of small round cells embedded in a cellular fibroblastic stroma is typical feature of DSRCT along with the dot-like immunostaining for desmin and negative for CK5/6 and calretinin. DSRCT appears to show multidirectional differentiation toward muscle, neural, and epithelial elements. (6) Small cell carcinoma of the lung (SCLC): It lacks a desmoplastic stroma and the tumor cells are negative to immunostaining for desmin and S100. Tuberculosis and sarcoidosis also need to be excluded as they may present with similar clinical features. NHL Small round cell variety is excluded by the negative immunostaining for LCA / Anti-CD45 of the tumor cells. ${ }^{5}$

According to the Ann Arbor Classification of NonHodgkin Lymphoma, NHL Stage IV indicates extensive (diffuse) involvement in one organ or site, with/without NHL in distant lymph nodes. In our case the right lung was diffusely involved by the malignancy along with patchy infiltration of the left lung, suggesting stage IV NHL. The patient had no systemic B symptoms such as fever (greater than $101.5^{\circ}$ ), 
drenching night sweats, unexplained weight loss of $10 \%$ or more within the last 6 months .In our case there was nodal and primarily extra -nodal involvement $(E)^{6}$. By adding the biologic grade of the disease the staging of our case is Stage IV E NHL intermediate grade(small round cell variety).

The treatment approach should be individualized according to the period of gestation, stage and localization of the disease, the presence or absence of B symptoms (fever, night sweats, and weight loss of more than $10 \%$ of the original weight six months prior to first attendance) and the progression of symptoms and signs. ${ }^{7}$ Almost all NHL in pregnancy is high grade and most rapid tumour growth is thought to occur in early pregnancy and puerperium especially during lactation. Tumour masses of non-Hodgkin's lymphoma greater than $10 \mathrm{~cm}$ in size or mediastinal mass occupying more than half of the transverse thoracic diameter that is bulky disease and raised serum LDH represent poor prognostic signs. ${ }^{1}$

Although both radiotherapy and chemotherapy are potentially teratogenic, they can be used safely in some circumstances during pregnancy. A variety of protocols of combination chemotherapy ( CT) has been used for the treatment of $\mathrm{NHL}$ in pregnancy in the reported cases with variant outcomes. These are CHOP, CHOP II (cyclophosphamide, vincristin, adriamycin.prednisolone), VACOP- B, CHOP with rituximab and last of all, autologus stem cell transplantation with high-dose CT and ESHAP protocol (Etoposide VP16, Cisplatin, methylprednisolone and ephosphomide) . ${ }^{7-11}$ Radiation with proper shielding can also be given above the diaphragm during the first trimester. Later on it can be used only in areas away from the foetus. ${ }^{3}$ Chemotherapy alone cures $30 \%$ to $40 \%$ of patients with advanced disease of Stage III or Stage IV. Two strategies for treating localized intermediate and highgrade Non-Hodgkin's Lymphoma has emerged without any convincing evidence in favour of either strategy over the recent decades: Chemotherapy alone with CHOP for 6-8 cycles, or a short course of Chemotherapy (usually 3 cycles of $\mathrm{CHOP}$ ) followed by involved - field Radiotherapy. The presumed advantages of chemotherapy alone are avoidance of long -term complications of radiotherapy and the higher total doses of systemic therapy which increases the potential for eliminating microscopic sites of disease. The possible benefits of short course
Chemotherapy followed by Radiotherapy are the reduction in the risk of cardiac toxicity due to the lower total dose of Doxorubicin, the use of two mainly cross-resistant treatments and the advantage of radiation directly to sites of detectable disease. ${ }^{12}$ In this case however chest radiotherapy was given after seven cycles of $\mathrm{CHOP}$ therapy for progressive disease and she did not suffer from any clinically or echocardiographically proven Doxorubicin -induced cardiac toxicity, may be because of short survival.

Children exposed to high-dose doxorubicincontaining combination chemotherapy in utero (especially during the second and third trimester) have been found to be normal subsequently, with follow-up ranging from several months to 11 years. There are no data regarding long-term effects on children exposed in utero for most of the chemotherapeutic agents used for treatment of $\mathrm{NHL} .{ }^{13} \mathrm{Up}$ to submission of the report of this case the child is in apparent good health.

\section{Conclusion:}

Lymphoma is a rare diagnosis in pregnancy. The diagnosis of $\mathrm{NHL}$ in pregnancy may be delayed because of reluctance to subject the patient to $X$ - rays and surgical procedures for the non-specific symptoms. Therefore this diagnostic delay should be avoided by all means maintaining a liaison between haematologist, oncologist and obstetrician. Immunohistochemical markers provide prognostic stratification and together with other clinical factors can configure the risk profile for certain groups of patients more accurately, with critical impact on therapeutic decisions. ${ }^{14}$ Therefore immunohistochemical evaluations should be considered in every case.

\section{Reference:}

1. Habib F.A. Pregnancy in a patient with nonHodgkin lymphomas: Qatar Medical journal 2002; 11(1).

2. Non-Hodgkin's Lymphoma During Pregnancy, General Information About Non-Hodgkin Lymphoma During Pregnancy. http://cancer.gov

3. Jesus Ortega. Multiple agent chemotherapy including bleomycin of non-hodgkin's lymphoma during pregnancy; Cancer cytopathology CA: A cancer journal for clinician. 40(6): 2829-2835 
4. Non-Hodgkin's lymphoma during pregnancy: Treatment - Health Professional Information: National Cancer Institute via the Internet web site at http://cancer.gov or call 1-800-4-CANCER

5. WANG Zhao-ming, XIAO Wen-bo, ZHENG Shusen Desmoplastic small round cell tumor of the lung: case report Chinese Medical Journal 2007;120(24):2327-2328

6. J । O Craig, D B L McClelland, C A Ludlam . Haemtological Malignancies. Davidson's Principles and practice of Medicine,20 e, Churchill Livingstone. P 1048

7. C.C. Lees, M. Tsirigotis, J.V.L. Carr and M.A. Richards' $T$ cell non-Hodgkin's Iymphoma presenting in the first trimester of pregnancy Postgrad Med J 1994; 70: 371-372.

8. Masuhiro Kazuo .Successful chemotherapy during pregnancy complicated with nonHodgkin's lymphoma. Advances In Obstetrics And Gynecology. 2001; 53(4): 323-328.

9. J.M. Rodriguez and M. Haggag. VACOP-B chemotherapy for high grade non-Hodgkin's lymphoma in pregnancy; Clinical oncology 1995; 7(5): 319-320.
10. Michael Herold, Sabine Schnohr, Hans Bittrich. Efficacy and Safety of a Combined Rituximab Chemotherapy during Pregnancy Journal of Clinical Oncology, 2001; 19(14): 34-39.

11. Pregnancies after high-dose chemotherapy and autologous stem cell transplantation ASCT in aggressive lymphomas. Blood, 2002; 100(2): 736-736.

12. Thomas P. Miller. Steve Dahlberg. Chemotherapy alone compared with Chemotherapy plus Radiotherpy for localized Intermediate and High grade Non-Hodgkin's Lymphoma.The new England Journal of Medicine 1998; 339(2): 2126.

13. Nantel S, Parboosingh J, Poon MC: Treatment of an aggressive non-Hodgkin's lymphoma during pregnancy with MACOP-B chemotherapy. Med Pediatr Oncol 1990; 18 (2): 143-145.

14. Cornelie Dudea. Study of clinical, anatomopathological and biological prognostic factors in Non-Hodgkin lymphoma. Prognostic parameters of NHL .pdf. 